\title{
Relato de Experiência do Estágio Multidisciplinar Interiorizado (EMI): Assistência ao Pré-Natal
}

Experience Report of the Multidisciplinary Stage Internalized (MSI): Prenatal Care

Experiência Informe de la Etapa Multidisciplinar Internalizado (EMI): Atención Pré Natal

Claudia Santos Martiniano ${ }^{1}$

Ardigleusa Alves Coelho ${ }^{2}$

Wezila Gonçalves do Nascimento ${ }^{3}$

Maria Valéria Beserra Cosme ${ }^{4}$

Maria Lúcia da Silva ${ }^{5}$

Jordy Alison de Lemos ${ }^{6}$

\section{Resumo}

Objetivo: Descrever a experiência vivenciada enquanto acadêmica de enfermagem, acerca da atuação do enfermeiro na assistência à gestante na Estratégia Saúde da Família em um município de pequeno porte do Estado da Paraíba. Método: Trata-se de um estudo de natureza descritiva tipo relato de experiência acerca da vivência no Estágio Multidisciplinar Interiorizado (EMI), sobre a atuação do Enfermeiro durante o pré-natal na Estratégia Saúde da Família, no município Alagoa Nova no período de 11 a 29 de Abril de 2016. Resultados: Foi possível observar a atuação do enfermeiro nas consultas de pré-natal de baixo risco. Durante as consultas eram realizados o exame físico, embora de forma incompleta em algumas consultas; interpretação de exames laboratoriais; prescrição de medicamentos; solicitação de exames; educação em saúde por meio de orientações gerais sobre a gestação e cuidados com o recém-nascido após o nascimento. As consultas eram alternadas entre a enfermeira e o médico. Quando havia intercorrência, a

${ }^{1}$ Doutora em Ciências da Saúde. Docente do Departamento de Enfermagem da UEPB. Autora correspondente: Rua Baraúnas, 351. Departamento de Enfermagem. Universitário, Campina Grande - PB. Brasil. 58429-500. E-mail: cmartiniano@ibest.com.br ${ }^{2}$ Doutora em Ciências da Saúde. Docente do Departamento de Enfermagem da UEPB.

${ }^{3}$ Enfermeira. Mestranda do Programa de Programa de Saúde Pública/UEPB. Estratégia Saúde da Família de Campina Grande-PB.

${ }^{4}$ Graduanda em Enfermagem pela (UEPB).

${ }^{5,6}$ Enfermeira(o). Hospital de Emergência e Trauma Dom Luiz Gonzaga Fernandes. 
gestante era encaminhada para atendimento médico. A assistência era prestada de forma humanizada e com equidade as mulheres no período gravídico de modo a proporcionar a detecção precoce de possíveis problemas que possam ocorrer durante a gestação. Conclusão: É preciso estar atento à consulta de pré-natal de forma completa mediante o grande leque de informação a serem registradas em vários impressos que envolvem essa consulta.

\section{Descritores: Atenção à Saúde; Saúde Materno-Infantil; Cuidado Pré-Natal.}

\section{Abstract}

The objective of this study is to describe the nursing experience as a nursing student about the nurse's role in assisting the pregnant woman in the Family Health Strategy in a small municipality in the state of Paraíba. It was possible to observe the nurse's performance in low-risk prenatal consultations. During the consultations the physical examination was performed, although incompletely in some consultations; Interpretation of laboratory tests; Prescription drugs; Examination request; Health education through general guidelines on gestation and newborn care after birth. The consultations were alternated between the nurse and the doctor. When there was an intercurrence, the pregnant woman was referred to the doctor. Assistance was provided in a humanized and fair manner to women in the pregnancy period in order to provide early detection of possible problems that may occur during gestation. However, it is necessary to be attentive to the prenatal consultation completely through the wide range of information to be recorded in several forms that involve this consultation.

\section{Descriptors: Health Care; Maternal and Child Health; Prenatal Care.}

\section{Resumen}

El objetivo es describir la experiencia vivida como una enfermería académica sobre el papel de la enfermera en la atención prenatal en la Estrategia Salud de la Familia en una pequeña ciudad en el estado de Paraiba. Fue posible observar el trabajo del personal de enfermería en las consultas prenatales de bajo riesgo. Durante las consultas se llevaron a cabo un examen físico, aunque de forma incompleta en 
algunas consultas; interpretación de las pruebas de laboratorio; medicamentos de prescripción; la solicitud de pruebas; educación para la salud a través de directrices generales sobre el embarazo y el cuidado del recién nacido después del nacimiento. Las consultas se alternan entre la enfermera y el médico. Cuando hubo complicaciones, la mujer embarazada fue remitido para su atención médica. Se prestó asistencia de una manera humana y con las mujeres durante la gestación de equidad para proporcionar una detección temprana de problemas potenciales que pueden ocurrir durante el embarazo. Sin embargo, uno debe estar atento a la consulta prenatal completamente a través de la amplia gama de información a registrar en varios impresos que implica esta consulta.

\section{Descriptores: Atención a la Salud; Salud Materno-Infantil; Atención Prenatal.}

\section{Introdução}

A Atenção Primaria de Saúde (APS) é compreendida como o primeiro contato do usuário com a rede de serviços, tendo como função coordenar o acesso e gerenciar o cuidado dentro de toda a rede de atenção ${ }^{(1-2)}$. No Brasil, a Estratégia Saúde da Família é o modelo proposto para a reorganização da Atenção Primária Saúde que permitiu maior acessibilidade, melhor satisfação dos usuários, bem como melhoria nas suas condições de saúde ${ }^{(1)}$.

No intuito de melhorar os indicadores relacionados a assistência a gestante, além da Estratégia Saúde da Família (ESF), implantada no Brasil desde 1994, inicialmente como programa, o Ministério da Saúde, no ano de 2000, implantou o Programa de Humanização do Pré-natal e Nascimento (PHPN) para regulamentar a assistência a gestante em todo Brasil. Desse modo, além de estabelecer o número de consultas ideais e a idade gestacional recomendada para o início do pré-natal já preconizada pelos protocolos da atenção primária, o programa institui os exames necessários para o acompanhamento seguro e a importância das ações de educação em saúde para assistência adequada ao binômio mãe-filho corroborando com as práticas assistências oferecidas em outros países ${ }^{(3)}$.

Em 2011, o Brasil implantou da Rede Cegonha com o intuito de colaborar com as ações desenvolvidas 
no PHPN. Além de todas as ações já realizadas, pretende-se garantir o acesso, acolhimento e a resolutividade dos serviços desde parto até os 24 meses de vida, oferecendo um cuidado humanizado, a testes e informações que lhe esclareçam sobre sua situação de saúde, planejamento familiar, doenças sexualmente transmissíveis, promovam saúde e previnam doenças e agravos com o objetivo de reduzir a mortalidade materna e infantil, com um olhar especial para a redução da mortalidade neonatal ${ }^{(4)}$.

Estudos relacionados ao número de consultas de pré-natal evidenciaram que o aumento do número de consultas está diretamente associado ao melhor resultado da gestação, que pode ser verificado com o melhor crescimento intrauterino, maior peso ao nascer, menor ocorrência de prematuridade ${ }^{(5)}$.

Nesse contexto, a ESF é responsável pelo primeiro contato da gestante com a rede de atenção devendo está capacitada para oferta de um prénatal de qualidade contribuindo para redução da morbimortalidade maternoinfantil e melhoria dos indicadores de saúde dessa população ${ }^{(6)}$.

$\mathrm{Na}$ gravidez de baixo risco à usuária deve ser acompanhada pela equipe saúde da família, enquanto nas de alto risco tem garantido $o$ acompanhamento simultâneo da equipe do centro de referência para gestantes de alto risco e da unidade básica. $\mathrm{O}$ acompanhamento do ciclo gravídicopuerperal inicia-se na gestação estendose até o puerpério, quando deve ser realizado a visita domiciliar pela equipe da ESF, onde mãe e recém-nascido terão a continuidade da assistência ${ }^{(7)}$.

O pré-natal pode ser entendido como acompanhamento que a gestante recebe desde a concepção do feto até o início do trabalho de parto. Durante este período a execução da educação em saúde pela equipe se faz de forma continuada e o enfermeiro por meio da consulta de enfermagem acompanha a gravidez, as modificações morfológicas e fisiológicas provocadas pela gestação, trabalho de parto, cuidados pós-natal e tudo aquilo que possa interferir na vida da gestante, do parceiro e a sua família ${ }^{(8)}$

A atuação do Enfermeiro na ESF durante a realização do pré-natal de baixo risco proporciona $\mathrm{o}$ desenvolvimento de medidas favoráveis que visam à abordagem apropriada às necessidades individuais de cada gestante permitindo o monitoramento da saúde e bem estar da gestante e do desenvolvimento fetal, viabilizando a 
detecção precoce de possíveis problemas $^{(9)}$.

O Enfermeiro deve desenvolver uma escuta qualificada sem preconceitos e julgamentos e o diálogo sincero, permitindo dessa maneira que a mulher aborde sobre questões que possam está provocando perturbações, dúvidas e angústias, além de suas necessidades físicas e socais, possibilitando assim, o fortalecimento do vínculo profissional-usuário, fazendo da consulta um momento singular, construída a partir das crenças, valores e cultura da mulher tornando-a protagonista de sua história ${ }^{(8)}$.

O Enfermeiro tem um papel essencial no acompanhamento da mulher no ciclo gravídico puerperal e sua atuação pode contribuir com a promoção da saúde das gestantes acolhendo-as desde o início da gravidez, prevenindo os riscos e complicações e se os riscos estiverem presentes, minimizá-los, assegurando, no fim da gestação, o nascimento de uma criança saudável.

Diante do exposto, o objetivo desse estudo é descrever a experiência vivenciada enquanto acadêmica de enfermagem, acerca da atuação do enfermeiro na assistência à gestante na Estratégia Saúde da Família em um município de pequeno porte do Estado da Paraíba.

\section{Método}

Trata-se de um estudo de natureza descritiva tipo relato de experiência acerca da vivência no Estágio Multidisciplinar Interiorizado (EMI), sobre a atuação do Enfermeiro durante o pré-natal na Estratégia Saúde da Família, no município Alagoa Nova no período de 11 a 29 de Abril de 2016.

Alagoa Nova está localizada na mesorregião do agreste paraibano, região de predomínio de policultura alimentícia e de pecuária extensiva de corte. Tem uma população de 19.681 habitantes. Apresenta Índice de Desenvolvimento Humano Municipal (IDHM) de 0,576, considerado baixo (IDHM entre 0,5 e 0,59) e entre 2000 e 2010, as dimensões que mais cresceram em termos absolutos foram à longevidade, seguida de renda e educação. Apesar de ser considerado baixo, o IDHM de Alagoa Nova está acima da média estadual nacional. Houve um incremento do envelhecimento populacional, redução da mortalidade infantil em $50 \%$ e a esperança de vida ao nascer aumentou 13,9 anos nas últimas duas décadas. O 
índice de Gini aumentou de 0,52 para 0,60 refletindo o grau da concentração de renda do município ${ }^{(10)}$.

Quanto à organização dos serviços de saúde, Alagoa Nova compõe um dos 70 municípios da $2^{\circ}$ Macrorregião de Saúde, polarizada por Campina Grande. Para o entendimento da rede que constitui da $2^{\circ}$ macrorregião, Alagoa Nova e mais 11 municípios formam a $9^{\circ}$ Região de Saúde denominada de Renascer do Brejo. A rede de assistência municipal formada pelos seguintes estabelecimentos de saúde: 1 unidade mista, 3 postos de saúde, 1 unidade móvel terrestre, 2 consultórios isolados, 7 centros de saúde de unidade básica ${ }^{(11)}$.

O estágio foi realizado em uma unidade (mista) localizada na zona urbana. A equipe de saúde era composta, por uma enfermeira, um odontólogo, uma técnica de higiene bucal (THB), uma médica e oito agentes comunitários.

\section{Resultados e Discussão}

Durante a vivência foram observadas 17 consultas de pré-natal, com mulheres na faixa etária 18 a 36 anos de idade, sendo 4 primigestas, 10 de secundigesta e 3 multíparas.
Em relação à idade gestacional, 6 estavam no $1^{\circ}$ trimestre, 5 no $2^{\circ}$ trimestre e 6 no terceiro trimestre. $\mathrm{Na}$ unidade de saúde, o agendamento das gestantes para o pré-natal está organizado segundo a idade gestacional, conforme preconiza o Ministério da Saúde, com consultas mensais até a $28^{\circ}$ semana, quinzenal da $28^{\circ}$ semana até a $36^{\circ}$ semana e semanal a partir da $37^{\circ}$ até o final da gestação ${ }^{(7)}$.

Para cada gestante que comparecia à primeira consulta foi realizado o preenchimento do cartão da gestante com o nome, idade, número do cadastro no Sistema Único de Saúde (SUS), endereço, naturalidade, antecedentes familiares e pessoais, cor, profissão, estado civil, condições de moradia e saneamento básico, calculada a idade gestacional (IG) e a data provável do parto e o cadastro no SISPRENATAL.

Em seguida era realizada a anamnese, o exame físico com a inspeção da pele, aferição da pressão arterial, peso, ausculta dos batimentos cardiofetais (BCF) com o sonar doppler, foi verificada a altura do fundo uterino e a presença ou não de edemas nos membros inferiores (MMII). A consulta teve continuidade com a escuta das queixas das gestantes com 
esclarecimentos de dúvidas, informações sobre a importância da realização dos exames, bem como a necessidade de tomar as vacinas, caso ela não estivesse imunizada. Observouse, porém, que a avaliação de mucosas, da tireóide, mamas, pulmões, coração e abdome não foram presenciadas.

Foram solicitados os exames laboratoriais do primeiro trimestre como: hemograma, grupo sanguíneo; fator RH; glicemia; hemograma; toxoplasmose; rubéola; realização teste rápido $\mathrm{HIV}$, sífilis e hepatite $\mathrm{B}$ e $\mathrm{C}$; citomegalovírus; VDRL; sumário de urina e ultrassonografia obstétrica. Vale ressaltar que as consultas eram alternadas entre a médica e a enfermeira, corroborando com as recomendações do Protocolo de Atenção ao Pré-natal de Baixo Risco.

Nas consultas subsequentes, no exame físico era realizada a aferição da pressão arterial, peso, ausculta dos batimentos cardiofetais, medição da altura do fundo uterino, cálculo da idade gestacional, interpretação de exames, o estado de imunização, presença de movimentos fetais, escuta das queixas e feito orientações gerais em relação ao ciclo gravídico puerperal. Durante a realização de cada consulta as gestantes tinham sua classificação de risco avaliada e se fosse necessário, a mesma era encaminhada imediatamente para avaliação médica.

Ressalta-se que a pesquisa de edema, o exame ginecológico e das mamas, para observação do mamilo não foram efetivados.

As gestantes a partir da $20^{a}$ semanas de gestação iniciavam a suplementação do ferro, porém em relação ao ácido fólico que deveria ser iniciado desde o planejamento da concepção até a décima segunda semana de gestação com objetivo de ajudar na formação do tubo neural ${ }^{(7)}$, não foi observado sua prescrição durante a assistência.

No terceiro trimestre de gestação eram solicitados novos exames laboratoriais. O acesso ao diagnóstico do HIV e sífilis é direito de toda gestante/parturiente ${ }^{(12)}$ e de acordo com o protocolo do pré-natal de baixo risco $^{(7)}$, recomenda-se a realização do teste com aconselhamento e consentimento, na primeira consulta de pré-natal e a repetição, no início do terceiro trimestre e dessa forma efetivar os pressupostos da Rede Cegonha, garantindo o acolhimento com avaliação do risco e da vulnerabilidade, bem como ampliação do acesso e melhoria do pré-natal $^{(13)}$. 
Nesse contexto, foram realizados testes rápidos em dez gestantes, sendo sete no primeiro e três no terceiro trimestre. Antes da realização do teste foi aplicado um breve questionário e em seguida eram realizados os testes para HIV, Sífilis, hepatite B e C. Durante a execução dos exames, todas as gestantes eram orientadas e incentivadas a usarem preservativos durante as relações sexuais.

O teste rápido para sífilis em uma das gestantes na $38^{\text {a }}$ semana obteve reagente como resultado, diferentemente do teste rápido realizado no primeiro trimestre que havia sido não reagente. Em virtude do resultado encontrado, a gestante foi orientada pela enfermeira e pela médica sobre os riscos da sífilis para mãe e para o feto, quando não tratada. Em seguida foi solicitado VDRL, em virtude de que para a confirmação do diagnóstico quantitativo é preferível, pois seus títulos correlacionam-se diretamente com a atividade da doença, se positivo a mesma iniciaria o tratamento o mais rápido possível, porém não ficou claro se o tratamento seria prescrito e instituído pelos profissionais da Unidade de Saúde ou a gestante seria encaminhada para algum Serviço de Referência.
Em relação ao processo de educação em saúde, durante as consultas, eram repassadas informações sobre sexualidade na gravidez, amamentação, sinais e sintomas do trabalho de parto, informações sobre as vantagens do parto normal tanto para a mãe quanto para o bebê, apoio psicológico, avaliação nutricional, exercícios para alivio das dores, cuidados com a saúde bucal ${ }^{(8)}$, todavia se faz necessário refletir a condução desse processo, pois o repasse de informações sem a construção coletiva entre os sujeitos envolvidos fragiliza ação, não sensibiliza e ainda não promove a transformação dos determinantes de saúde e doença. O prénatal é um momento oportuno para que as mulheres possam se cuidar $\mathrm{e}$ contribuir para uma gestação saudável e redução de risco e complicações.

Foi possível perceber a atuação da Enfermeira como educadora em saúde tanto nas consultas individuais quanto no grupo de gestantes. E isso gera um laço de confiança entre as gestantes e a profissional.

Em todas as consultas realizadas foi possível observar um amplo registro de informações em vários impressos, tais como o cadastro do SISPRENATAL ou a ficha de 
seguimento, o cartão da gestante, o prontuário da gestante e ainda a ficha de atendimento do e-SUS. Esse excesso de informação, embora altamente necessários, pode levar o profissional ao aligeiramento das consultas, menosprezando aspectos do exame físico, como foi observado.

\section{Conclusão}

Durante o EMI, foi possível notar o quanto a consulta de pré-natal é importante para redução de complicações, identificação de risco e consequentemente redução da mortalidade materno e infantil. Foi muito gratificante acompanhar a gestante durante sua gravidez e saber que o parto e o puerpério ocorreram bem, graças à realização de um prénatal de qualidade e humanizado.

\section{A vivência possibilitou a} oportunidade de aprender como fazer uma consulta pré-natal, classificar o risco da gestação, adquirir conhecimento em educação em saúde, como também a realização do exame físico na gestante, e o principal, aprender que uma boa assistência ao pré-natal só e possível com a escuta qualificada, de forma crítica e reflexiva, das queixas, anseios, medos e dúvidas da usuária.

Chama atenção a quantidade de registros realizados em vários impressos durante a consulta de pré-natal, que embora necessário, pode conduzir a incompletude do exame físico ou outras ações importantes na consulta. Depreende-se daí a necessidade de equilibrar a demanda agendada de modo a se obter tempo necessário à consulta de qualidade.

\section{Referências}

1. Ministério da Saúde (BR). Starfield B. Atenção Primária: equilíbrio entre necessidades de saúde, serviços e tecnologia. Brasília: Unesco/Ministério da Saúde; 2002.

2. Organização Mundial de Saúde (OMS). Relatório mundial de saúde 2008: cuidados de saúde primários agora mais que nunca. Lisboa: Organização Mundial da Saúde e Alto Comissariado para a Saúde; 2008.

3. Ministério da Saúde (BR). Portaria GM no. 569/2000. Institui o Programa Pré-natal e Nascimento. Brasília: Ministério da Saúde; 2000.

4. Martinelli et al. Adequação do processo da assistência pré-natal segundo os critérios do Programa de Humanização do Pré-natal e Nascimento e Rede Cegonha. Rev Bras Ginecol Obstet. 2014; 36(2):56-6. 
5. Ribeiro ER, Guimarães AM, Bettiol H, Lima DD, Almeida ML, Souza L, et al. Risk factors for inadequate prenatal care use in the metropolitan area of Aracaju, Northeast Brazil. BMC Pregnancy Childbirth. 2009; 9:31.

6. Ministério da Saúde (BR). Secretaria de atenção à saúde. Protocolo de atenção à saúde e resposta à ocorrência de microcefalia relacionada à infecção pelo vírus zika. Brasília: Ministério da Saúde; 2015.

7. Ministério da Saúde (BR). Secretaria de atenção à saúde. Departamento de atenção básica. Atenção ao pré-natal de baixo risco. Brasília: Ministério da Saúde; 2013.

8. Barbosa RKL. Gravidez, sexualidade e importância do enfermeiro no prénatal: análise do discurso da literatura. Monografia (Graduação). Paraíba: Universidade Estadual da Paraíba Campina Grande-PB; 2012:38.

9. Quilo JD. Assistência pré-natal: uma vivência positiva durante o estágio multidisciplinar interiorizado (EMI). Monografia (graduação). Paraíba: Universidade Estadual da Paraíba. Campina Grande- PB; 2014:29.

10. Instituto Brasileiro de Geografia e Estatística (IBGE). Censo 2010. Rio de Janeiro: IBGE; 2010.

11. Secretaria de Estado da Saúde da Paraíba (PB). Plano Diretor de Regionalização. Paraíba: Secretaria de Estado da Saúde; 2008. Disponível em: $<$ http://www.saude.pb.gov.br/site/PDR 08.pdf>. Acesso em: 29 de out. 2016.
12. Silva O, Tavares LHL, Paz LC. As atuações de enfermeiro relacionadas a tetes rápido anti-HIV diagnóstico: uma reflexão de interesse da enfermagem e da saúde pública. Rev. Enfermagem em Foco. 2011; 2 (supl):58-62.

13. Ministério da Saúde (BR). Secretaria de Vigilância em Saúde Departamento de DST/Aids e Hepatites Virais. Realização do Teste Rápido para HIV e Sífilis na Atenção Básica e Aconselhamento em DST/Aids. Brasília: Ministério da Saúde; 2012. 